\title{
A CXCR4 antagonist leads to tumor suppression by activation of immune cells in a leukemia-induced microenvironment
}

\author{
A-REUM HAN ${ }^{1}$, JI YOON LEE ${ }^{1,5}$, HEE-JE KIM ${ }^{1,2}$, WOO-SUNG MIN ${ }^{2}$, \\ GYEONGSIN PARK $^{1,3}$ and SE-HOON KIM ${ }^{1,4}$ \\ ${ }^{1}$ Cancer Research Institute, Seoul St. Mary's Hospital; ${ }^{2}$ Department of Hematology, Catholic Blood and \\ Marrow Transplantation Center, College of Medicine, The Catholic University of Korea, Seoul; \\ ${ }^{3}$ Department of Pathology, College of Medicine, The Catholic University of Korea, Seoul; \\ ${ }^{4}$ Daewon Foreign Language High School, Seoul, Republic of Korea
}

Received June 24, 2015; Accepted August 28, 2015

DOI: 10.3892/or.2015.4297

\begin{abstract}
The bone marrow microenvironment (BMM) provides a protective niche that supports growth and survival of normal and leukemic hematopoietic stem cells. The SDF-1/CXCR4 interaction is critical for regulation of homing to and retention of hematopoietic cells in the bone marrow (BM), which leads to increased chemoresistance. SDF-1/ CXCR4 plays pivotal roles in cross-interactions between blasts and the BMM to prevent retention and mobilization of leukemic cells, as well as in normal hematopoiesis including the development of immune cells. We show that the CXCR4 antagonist, plerixafor, decreased the level of CXCR4 expression and inhibited SDF-1-induced migration of leukemic cells. Further, the inhibition of the interaction between leukemic cells and the BMM by the plerixafor enhanced cytotoxic activity of immune cells as a result of increased susceptibility of leukemic cells to chemotherapeutic agents such as cytosine arabinoside (Ara-C) in a mouse model of acute myeloid leukemia (AML), suggesting biological effects of the BMM through immune cell activation. Because alterations in the BMM promote retention and survival of leukemic cells, targeting the niche is regarded as an advanced strategy to eradicate drug-resistant leukemic blasts. This study demonstrates that the effects of CXCR4 inhibition on blast suppression and immune cell function in the tumor microenvironment and chemotherapy
\end{abstract}

Correspondence to: Professor Hee-Je Kim, Division of Hematology, Department of Internal Medicine, Cancer Research Institute, Catholic Blood and Marrow Transplantation Center, Seoul St. Mary's Hospital, College of Medicine, The Catholic University of Korea, 222 Banpo-daero, Seocho-gu, Seoul 137-701, Republic of Korea

E-mail: cumckim@catholic.ac.kr

Present address: ${ }^{5}$ Yonsei University College of Medicine, Seoul, South Korea

Key words: acute myeloid leukemia, tumor microenvironment, leukemic cells, immune cells, CXCR4 with plerixafor represents an advanced therapeutic strategy of targeting the leukemic niche.

\section{Introduction}

Acute myeloid leukemia (AML) is defined by an increase in undifferentiated myeloid cells in bone marrow (BM) with abnormal genetic changes, resulting in hematopoietic insufficiency (1). The cells of most leukemias, including AML, interact with the BM microenvironment (BMM), which influences their survival (2). In particular, leukemic blasts after chemotherapy are protected by factors in tumor environments and this may result in a relapse in leukemia. Because alterations in the BMM promote retention and survival of leukemic cells, targeting this niche is regarded as an advanced strategy to eradicate drug-resistant leukemic blasts $(3,4)$. The interaction between hematopoietic cells and the BMM through various factors is critical for regulation of cell homing, proliferation, and differentiation. Several studies have reported that BM stromal cells protect leukemic cells from chemotherapy-induced apoptosis $(5,6)$. Among many factors in the BMM, immune cells participate in crosstalk with hematopoietic stem cells (HSC) (7). The chemokine CXC motif ligand 12 (CXCL12), also known as stromal-derived factor-1 (SDF-1), is a strong attractant that recruits $\mathrm{CXC}$ receptor 4 (CXCR4)-expressing hematopoietic cells to BM. The interaction of SDF-1/ CXCR4 plays pivotal roles in the cross-interaction between blasts and the BMM to prevent retention and mobilization of leukemic cells (8), as well as in normal hematopoiesis including the development of immune cells $(9,10)$. Plerixafor $\left(\right.$ Mozobil $^{\mathrm{TM}}$ ) rapidly induces mobilization of hematopoietic stem cells from BM into peripheral circulation by blocking SDF-1/CXCR4 $(11,12)$. Because most primary AML retains a dependency on the BMM, plerixafor can function as a microenvironmental factor in leukemia, decreasing the resistance of leukemic cells by modulating the BMM and directly suppressing malignancies through modulating the expression of CXCR4 $(13,14)$. The correlation between upregulation of CXCR4 after chemotherapy and poor outcomes has been shown by Sison et al (15). In addition, the inhibition of SDF-1/CXCR4 has been shown to enhance the sensitivity of 
leukemic cells to chemotherapeutic agents, as well as partly abolish their protection by the BMM $(13,16)$. Based on these studies, we examined whether CXCR4 inhibition by plerixafor could activate the killing function of immune cells, resulting in the suppression of blasts in vivo. To answer this question, an established syngeneic leukemic mouse model using C1498 cells (a murine myelogenous leukemia cell line) was used (17). We found that plerixafor did not eradicate the leukemic blasts in vitro; however, it made leukemic blasts more sensitive to cytotoxic chemotherapy with cytosine arabinoside (Ara-C) in vivo, suggesting biological effects on the microenvironment through immune cell activation. Furthermore, we report an additional role for CXCR4 inhibition in tumors as a stimulator of immune cells, except migration capacity. The present study shows that CXCR4 inhibition induces the suppression of AML blasts with chemotherapy by the upregulation of cytokines to kill the cancerous cells. Further, it provides some clues to develop therapeutic strategies involving immune cell activation in the leukemic microenvironment.

\section{Materials and methods}

Human primary cells and cell lines. All experiments were performed with authorization from the Institutional Review Board for Human Research at the Catholic University of Korea. AML blood samples were obtained from the Catholic Blood and Marrow Transplantation Center at Seoul St. Mary's Hospital. A total of 19 AML samples were prospectively collected and examined. Mononuclear cells were separated from leukapheresed peripheral blood (LPB) by density gradient centrifugation using Ficoll Paque ${ }^{\mathrm{TM}}$ Plus (17-1440-03; GE Healthcare Life Sciences, Piscataway, NJ, USA). The clinical characteristics and experimental information of the AML patients enrolled in the present study are listed in Table I. The human AML cell line, Jurkat (TIB-152 ${ }^{\mathrm{TM}}$; American Type Culture Collection, ATCC), and the murine AML cell line, C1498 (a murine myelogenous leukemia cell line, TIB-49 ${ }^{\text {тм }}$; ATCC), were used. All cells were cultured in the proper media at $37^{\circ} \mathrm{C}$ in a humidified atmosphere of $5 \% \mathrm{CO}_{2}$, according to the supplier's suggestions.

Leukemic mouse model. C57B1/6J mice were purchased from the Jackson Laboratory (Bar Harbor, ME, USA) and bred under pathogen-free conditions in the Department of Laboratory Animals at the Catholic University of Korea. All of the animal experiments were approved by the Institutional Animal Care and Use Committee of the Catholic University of Korea. For the syngeneic model, $2 \times 10^{6} \mathrm{C} 1498$ cells were suspended in $200 \mu \mathrm{l}$ phosphate-buffered saline (PBS) and were intravenously injected into the 7-week-old mice. For the treatment, plerixafor (Mozobil ${ }^{\mathrm{TM}}, 2.5 \mathrm{mg} / \mathrm{kg}$; Sanofi Oncology) were subcutaneously injected $2 \mathrm{~h}$ before and $2 \mathrm{~h}$ after intraperitoneal injection of Ara-C (100 mg/kg; Sigma) into the mice administered C1498 cells, as described in Fig. 3A (schematic diagram). Peripheral blood (PB) was obtained from a facial vein and samples from organs were obtained from the sacrificed mice until day 30 post-injection.

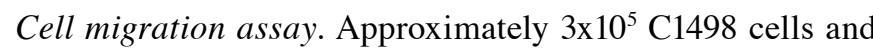
human primary cells were pretreated with serially diluted plerixafor in DMEM with $10 \%$ fetal bovine serum (FBS) for $2 \mathrm{~h}$. After washing, total cells were resuspended in $500 \mu \mathrm{l}$ of Dulbecco's modified Eagle's medium (DMEM) with 1\% FBS and placed in the upper chamber of Transwell plates $(3 \mu \mathrm{m}$ membrane pore size; Corning). Inserts were placed in the lower chamber containing the same medium, with or without SDF-1 $\alpha$ (100 ng/ml; R\&D Systems, Minneapolis, MN, USA). Migration assays were performed at $37^{\circ} \mathrm{C}$ for $4 \mathrm{~h}$. The migrated cells in the lower chamber were counted by an automated cell counter $\left(\right.$ Luna $^{\mathrm{TM}}$, LB-L10001).

Cell apoptosis assay. $\mathrm{C} 1498$ cells $\left(2 \times 10^{5}\right)$ were pretreated in 0 or $5 \mu \mathrm{M}$ CXCR4, plerixafor, in DMEM with $10 \%$ FBS at $37^{\circ} \mathrm{C}$ for $2 \mathrm{~h}$. Then, cells were cultured for $24 \mathrm{~h}$ at $37^{\circ} \mathrm{C}$ in DMEM with $10 \%$ FBS with or without Ara-C (40 ng/ml) and SDF-1 $\alpha(100 \mathrm{ng} / \mathrm{ml})$. Annexin $\mathrm{V}^{+}$apoptotic cells were stained (ApoScan; BioBud) and were counted by flow cytometry.

Flow cytometry. PB, spleen and BM cells were flushed from mouse femurs, suspended in $200 \mu \mathrm{l}$ of PBS, and incubated with antibodies. After washing, cells were analyzed using a FACSCalibur flow cytometer equipped with CellQuest ${ }^{\circledR}$ software (BD Biosciences, San Diego, CA, USA). The antibodies used to detect mouse cells included FITC-conjugated anti-mouse CD4 (clone: GK1.5, 553729), PE-Cy ${ }^{\mathrm{TM}}$ 5-conjugated anti-mouse CD8 (clone: 53-6.7, 553034), and PE-conjugated anti-mouse NK1.1 (clone: PK136, 553165) (all from BD Pharmingen $^{\mathrm{TM}}$ ), biotin-conjugated anti-mouse CXCR4 (clone: REA107, 130-102-021; Miltenyi Biotec), APC-conjugated streptavidin (17-4317-82; eBioscience), for human cells, APC-conjugated anti-human CXCR4 (clone: 12G5, 555976; BD Pharmingen $^{\mathrm{TM}}$ ). Flow cytometric data were analyzed using appropriate controls with proper isotype-matched IgG and unstained controls.

Histology. Liver, spleen, and BM from the each group were fixed in $4 \%$ paraformaldehyde. BM samples were fixed in paraformadehyde, decalcified with $5 \%$ formic acid, and embedded in paraffin. Prepared slides were counterstained with Meyer's hematoxylin. Hematoxylin and eosin (H\&E) staining was used after fixation to confirm leukemic blast infiltration in tissues including BM, spleen, and liver. For immunohistochemistry, after antigen retrieval, prepared slides were blocked for endogenous peroxidase activity and were incubated with primary antibody anti-mouse IFN- $\gamma$ (clone: DB-1, NB100-78214; Novus Biologicals). Proper secondary antibody (IH-8056-50; Gentaur, Brussels, Belgium) was used for immunohistochemistry and detected using the DAB chromogen/substrate system (HistoMouse ${ }^{\mathrm{TM}}-\mathrm{MAX}$ kit, 89-9551; Invitrogen, Camarillo, CA, USA). Slides were counterstained with Meyer's hematoxylin.

Quantitative real-time PCR ( $R T$ - $q P C R)$. Total RNA was extracted from BM cells, liver, and spleen of mice as described in the schematic diagram (Fig. 3A) at day 15. RNA $(1 \mu \mathrm{g})$ was reverse transcribed into cDNA at $42^{\circ} \mathrm{C}$ for $60 \mathrm{~min}$ in a 20- $\mu 1$ reaction mixture using a Transcriptor First Strand cDNA Synthesis kit (04 897030 001; Roche, Mannheim, Germany). The primers used in the study are listed in Table II. The RT-qPCR was performed with TaqMan probes by 
Table I. Clinical and laboratory features of the primary AML cells.

\begin{tabular}{|c|c|c|c|c|c|c|}
\hline Patients & FAB subtype & $\begin{array}{c}\text { Age at } \\
\text { diagnosis }\end{array}$ & Gender & Cell source & $\begin{array}{l}\mathrm{WBC} / \mathrm{mm}^{3} \\
\text { at } \operatorname{diagnosis}\end{array}$ & $\begin{array}{l}\text { Cytogenetic } \\
\text { anomalies }\end{array}$ \\
\hline 1 & M3 & 53 & M & PB & 114,980 & $46, X Y, t(15 ; 17)(q 22 ; q 12)[20]$ \\
\hline 2 & M4 & 65 & M & PB & 185230 & $46, X Y[20]$ \\
\hline 3 & M4 & 41 & $\mathrm{~F}$ & PB & 100260 & $46, X X, t(6 ; 11)(q 27 ; q 23)[30]$ \\
\hline 4 & M4 & 23 & $\mathrm{~F}$ & PB & 177420 & $\begin{array}{l}48 \sim 49, X X,+1, \operatorname{der}(1 ; 14)(\mathrm{q} 10 ; \mathrm{q} 10) \\
\mathrm{t}(7 ; 11)(\mathrm{q} 32 ; \mathrm{p} 15),+8,+8, \mathrm{t}(9 ; 11) \\
(\mathrm{p} 22 ; \mathrm{q} 23), \mathrm{t}(12 ; 20)(\mathrm{q} 12 ; \mathrm{q} 13.1), \\
\operatorname{del}(19)(\mathrm{p} 13.1),+\operatorname{mar}[\mathrm{cp} 2] / 46, \mathrm{XX}[28]\end{array}$ \\
\hline 5 & M1 & 48 & M & PB & 115550 & 47,XY,+11[29]/46,XY[1] \\
\hline 6 & M5 & 27 & M & PB & 40 & $46, X Y[20]$ \\
\hline 7 & M3 & 32 & $\mathrm{~F}$ & PB & 53.98 & $46, X X, t(15 ; 17)(q 22 ; q 12)[28] / 46, X X[2]$ \\
\hline 8 & M3 & 28 & M & PB & 121900 & $46, X Y[20]$ \\
\hline 9 & M1 & 50 & $\mathrm{~F}$ & $\mathrm{~PB}$ & 123140 & $46, X X, 15 p s+[20]$ \\
\hline 10 & M5b & 41 & $\mathrm{~F}$ & PB & 143720 & $46, X X, t(6 ; 11)(q 27 ; q 23)[20]$ \\
\hline 11 & M5 & 82 & $\mathrm{~F}$ & PB & 109540 & $46, X X[20]$ \\
\hline 12 & M4 & 39 & M & PB & 141200 & 47,XY,+mar[3]/46,XY[22] \\
\hline 13 & M2 & 25 & M & $\mathrm{PB}$ & 185000 & 46,XY[20] \\
\hline 14 & M4 & 45 & M & PB & 71820 & 46,XY,inv(16)(p13.1q22)[20] \\
\hline 15 & M5b & 28 & $\mathrm{~F}$ & PB & 128020 & $46, X X, t(6 ; 11)(q 27 ; q 23)[19] / 46, X X[1]$ \\
\hline 16 & M4 & 36 & $\mathrm{~F}$ & PB & 240640 & $46, X X[20]$ \\
\hline 17 & M0 & 58 & M & PB & 317620 & $46, X Y[20]$ \\
\hline 18 & M1 & 79 & $\mathrm{~F}$ & PB & 207970 & $46, X X, t(11 ; 12)(p 15 ; q 13), \operatorname{del}(3)(q 12 q 22)[20]$ \\
\hline 19 & M2 & 31 & $\mathrm{~F}$ & PB & 129700 & $46, \mathrm{XX}[20]$ \\
\hline
\end{tabular}

AML, acute myeloid leukemia; FAB, French American British; WBC, white blood cell; PB, peripheral blood.

Table II. Primer sequences for RT-PCR and RT-qPCR.

\begin{tabular}{lll}
\hline Gene & \multicolumn{1}{c}{ Sequence } & Method \\
\hline Mouse & F: TACCTCGCTATTGTCCACGC & RT-PCR \\
CXCR4 & R: GTGCACGATGCTCTCGAAGT & \\
Mouse & F: CGTGTTCCTACCCCCAATGT & \\
GAPDH & R: GGCCCTCAGATGCCTGCTTCAC & \\
Mouse & F: CAGCCGATGGGTTGTACCTT & RT-qPCR \\
IFN- $\gamma$ & R: GGCAGCCTTGTCCCTTGA & \\
& P: TGAGCTCATCCGAGTGGTCC & \\
Mouse & F: GACTGCTGCCCACGACAGA & \\
perforin & R: TGCCCGGAAATTGTTACC & \\
& P: CTTGGCCCATTTGG & \\
Mouse & F: CCCAGGCGCAATGTCAAT & \\
granzyme B & R: CCCCAACCAGCCACATAGC & \\
& P: TGAAGCCAGGAGATGTG & \\
Mouse & F: CGTGTTCCTACCCCCAATGT & \\
GAPDH & R: TGTCATCATACTTGGCAGGTTTCT & \\
& P: TCGTGGATCTGACGTGCCGC &
\end{tabular}

Direction: F, forward; R, reverse; P, probe.

LightCycler ${ }^{\circledR} 480$ (Roche). All data were normalized to the amount of GAPDH expression, with samples run in triplicate.
Statistical analysis. The results are presented as the mean \pm standard error (SE). Data were compared by the Mann-Whitney U test, and GraphPad Prism ver. 4 software (GraphPad Software, La Jolla, CA, USA) was used for the analyses. Image $\mathbf{J}$ was used for analysis of stained cells in slides. Percentage of stained area was calculated as the ratio of the stained area to the total area detected in the image. Values of $\mathrm{P}<0.05$ were considered statistically significant.

\section{Results}

Levels of CXCR4 are decreased in plerixafor-treated leukemic cells. To examine the level of CXCR4 expressed by AML blasts and murine cells, 19 AML samples and murine C1498 and human Jurkat cells were subjected to FACS analysis. Data revealed that both normal human samples and AML mononuclear cells highly expressed CXCR4. CXCR4 expression in both groups was significantly decreased by CXCR4 inhibition, when treated with $5 \mu \mathrm{M}$ plerixafor (normal, $79.7 \pm 14.2 \%$;

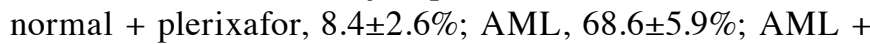
plerixafor, 30.8 $\pm 7.2 \%$; Fig. 1A). In addition, CXCR4 expression in C1498 cells was $\sim 31.29 \%$, compared to $99.7 \%$ in the CXCR4 + Jurkat cell line and RNA expression was also confirmed (Fig. 1B and C).

Plerixafor inhibits SDF-1 $\alpha$-induced migration of leukemic cells, but has no effect on cell apoptosis in vitro. The role of 


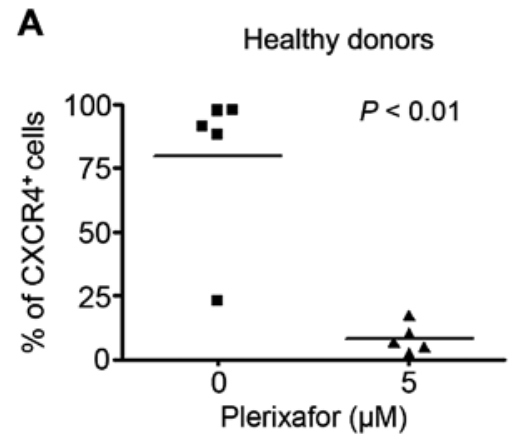

B

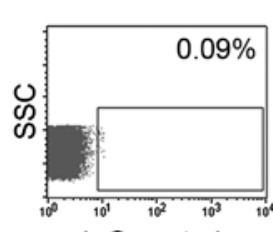

IgG control

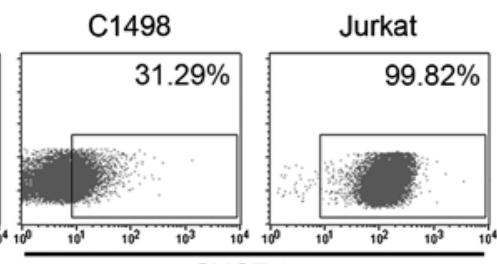

CXCR4

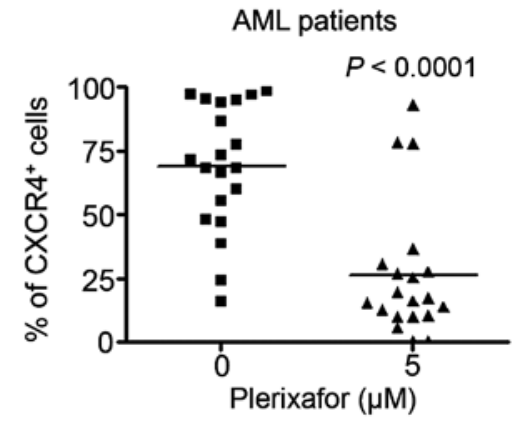

C

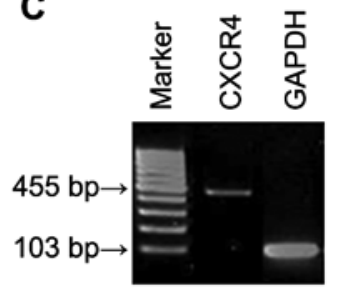

Figure 1. CXCR4 is expressed in human cells and decreased by plerixafor in normal healthy donors and primary AML blasts. (A) Both normal and blast cells exhibited a significant reduction in CXCR4 expression following plerixafor treatment. Data shown represents the mean of independent experiments (normal, $n=5$; AML, $n=19$ ). Bars in (A) represent mean \pm SE, and the statistical difference is presented in figures, compared to plerixafor treatment. (B) CXCR4 protein was clearly detected in C1498 cells by flow cytometry. The Jurkat cell line was used as a positive control. (C) RT-qPCR data show CXCR4 transcripts in C1498 cells.

CXCR4 in migration is well known. Osteoblasts and mesenchymal stromal cells (MSCs) in the BM niche produce cytokine SDF-1 $\alpha$, which is an attractant for CXCR4 + hematopoietic cells and encourages their migration. Because tumor cell migration into BM caused the recurrence of cancer in patients, we tested whether CXCR4 inhibition can block migration of C1498 cells under SDF-1 $\alpha$ exposure. Consistent with previous studies (14), our migration assay clearly showed the inhibitory effect of plerixafor on SDF-1 $\alpha$-induced migration of primary AML and C1498 cells. Under SDF-1 $\alpha$ conditions (100 ng/ml), C1498 cells were co-cultured with the plerixafor at various concentrations. Migrations of both $\mathrm{C} 1498$ and primary AML cells were similarly inhibited. While $42.8 \times 10^{2} \pm 18.1 \times 10^{2}$ migrated cells were detected without SDF-1 $\alpha$, SDF- $1 \alpha$ induced $140.6 \times 10^{2} \pm 48 \times 10^{2}$ cells to migrate, showing an $\sim 3.28$-fold increase. Although there is individual variation in primary AML cells, plerixafor significantly inhibited migration at all indicated doses. Similarly, in C1498 cells, cell migration was significantly inhibited by plerixafor in a dose-dependent manner (Fig. 2A). Primary AML cells were counted to avoid the induction of apoptosis during the experiments (Fig. 2B). As expected, plerixafor effectively inhibited tumor cell migration in vitro, confirming the functional role of the SDF-1/CXCR4 interaction in AML migration. Next, although the antileukemic effects by the plerixafor are still debatable, studies continue to show that SDF-1/CXCR4 can protect AML blasts from chemotherapy and induce the apoptotic machinery of leukemic blasts under BMM disruption $(8,18)$. Thus, to test the direct role of plerixafor in apoptosis, $\mathrm{C} 1498$ cells were cultured with or without Ara-C. At $24 \mathrm{~h}$ after co-culture, a FACS analysis was performed to evaluate the frequency of dead cells. A high number of apoptotic cells were observed following Ara-C treatment, compared to no apoptotic cells without Ara-C treat- ment. However, the Ara-C and plerixafor dual-treated group (termed $\mathrm{P}+\mathrm{A}$ group) displayed no significant difference in apoptosis when compared to the Ara-C only group (without SDF-1 $\alpha$ group: Plerixafor Ara- $\mathrm{C}^{-}$cells, $3.6 \pm 0.1 \%$; plerixafor $^{+}$ Ara- $\mathrm{C}^{-}$cells, $3.2 \pm 0.2 \%$; plerixafor $\mathrm{Ara}^{-} \mathrm{C}^{+}$cells, $18.4 \pm 2.5 \%$; plerixafor ${ }^{+}$Ara- $\mathrm{C}^{+}$cells, $16.0 \pm 1.3 \%$; and with $\mathrm{SDF}-1 \alpha$ group: plerixafor ${ }^{-}$Ara- $\mathrm{C}^{-}$cells, $3.3 \pm 0.3 \%$; plerixafor ${ }^{+} \mathrm{Ara}-\mathrm{C}^{-}$cells, $3.8 \pm 0.3 \%$; plerixafor ${ }^{-}$Ara- $\mathrm{C}^{+}$cells, 20.0 $\pm 2.0 \%$; plerixafor ${ }^{+}$Ara- $\mathrm{C}^{+}$cells, 17.7 $\pm 1.3 \%$; Fig. 2C). Dual treatment, therefore, does not increase leukemic blast deaths, suggesting that apoptosis is exclusively controlled by Ara-C, but not plerixafor, in vitro.

Significant suppression of blasts was detected in the plerixafor and Ara-C combination group in vivo. Because an in vitro system cannot recapitulate in vivo conditions, a syngeneic mouse model was used to further investigate the effects of plerixafor on blast suppression in a leukemic microenvironment. The protocol shown in Fig. 3A was used in experiments in vivo with tissues including liver, BM, and spleen from each group that were prepared and subjected to immunohistochemistry one month after C1498 injection. Results clearly displayed blast suppression in all tissues. Aberrant spindle-shaped C1498 cells distinguished these cells from normal HSC in BM, and leukemic clusters in the liver and spleen were detected. Leukemic blasts synergistically and significantly decreased in the $\mathrm{P}+\mathrm{A}$ group, compared to those of the other groups (Fig. 3B). It also provided quantificational significance in $\mathrm{P}+\mathrm{A}$ group, comparing the results to other groups (Fig. 3C) showing the following: In the BM control: $82.1 \pm 3.5 \%$; plerixafor, $65.7 \pm 3.8 \%$; Ara-C, $50.4 \pm 4.5 \%$; and $\mathrm{P}+\mathrm{A}, 36.1 \pm 4.4 \%$. In the liver control, $67.4 \pm 4.6 \%$; plerixafor, $58.2 \pm 2.5 \%$; Ara-C, 58.7 $\pm 2.9 \%$; and $\mathrm{P}+\mathrm{A}, 23.1 \pm 2.8 \%$. In the 


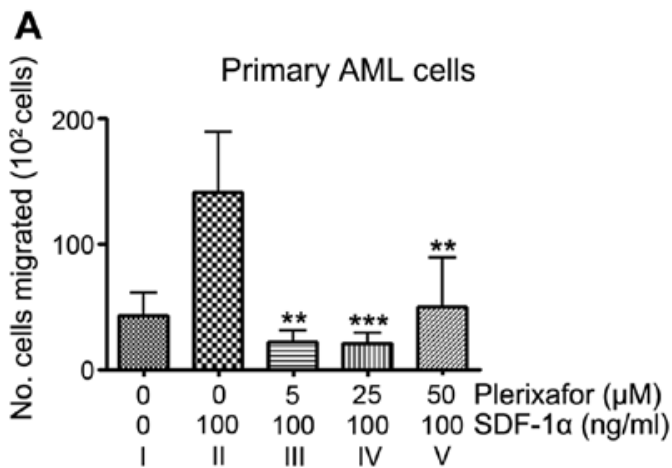

B

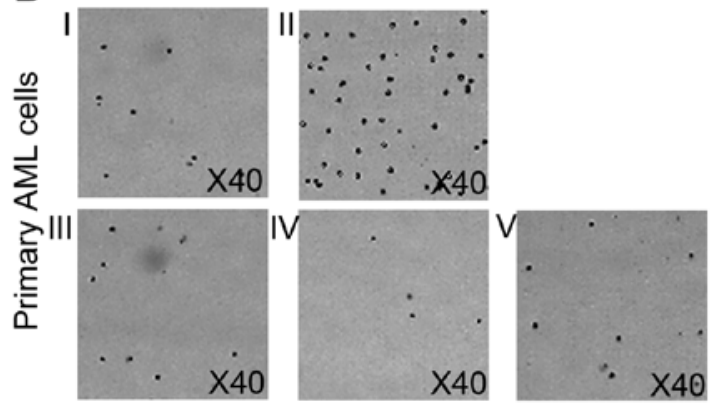

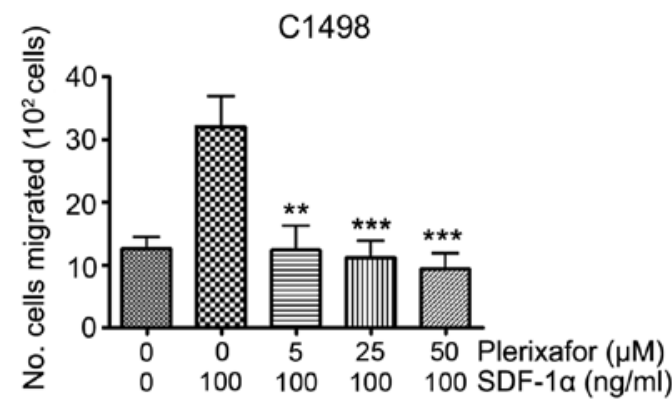

C

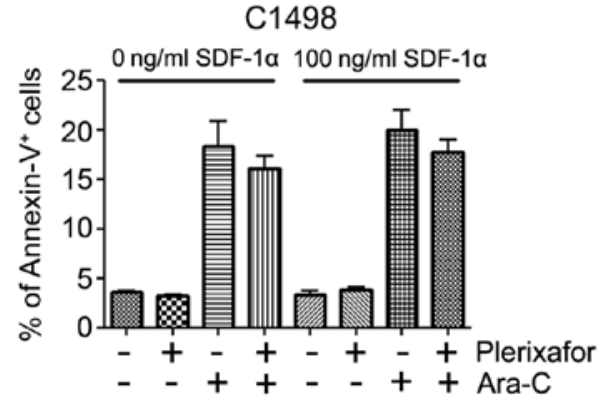

Figure 2. Plerixafor inhibits cell migration with SDF-1 $\alpha$ exposure in both primary AML cells and C1498 cells. (A) Migrated primary AML cells and C1498 cells were significantly decreased following plerixafor treatment under SDF-1 $\alpha$ induction. Data shown represents the means of independent experiments (AML, $n=8 ; C 1498, n=3$ ). Bars in (A) represent mean \pm SE, and asterisks depict a statistically significant difference in comparison with no plerixafor treatment. $\left({ }^{* *} \mathrm{P}<0.01 ;{ }^{* * *} \mathrm{P}<0.001\right)$. (B) Representative panels show migrated human blasts in the lower chambers in (A). I, plerixafor and SDF-1 $\alpha$ no treatment; II, plerixafor $0 \mu \mathrm{M}$ and SDF-1 $\alpha 100 \mu \mathrm{g} / \mathrm{ml}$; III, plerixafor $5 \mu \mathrm{M}$ and SDF-1 $\alpha 100 \mu \mathrm{g} / \mathrm{ml}$; IV, plerixafor $25 \mu \mathrm{M}$ and SDF-1 $\alpha 100 \mu \mathrm{g} / \mathrm{ml}$; V, plerixafor 50 $\mu \mathrm{M}$ and SDF-1 $\alpha$ $100 \mu \mathrm{g} / \mathrm{ml}$ (magnification, $\mathrm{x} 40$ ). (C) No difference in apoptosis by plerixafor treatment was detected in vitro.

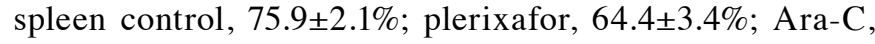
$47.4 \pm 3.5 \%$; and $\mathrm{P}+\mathrm{A}, 26.0 \pm 2.7 \%$. These data suggested an unexpected role for plerixafor in leukemic blast suppression specifically in the AML niche.

The frequency of immune cells was not increased; however, cytotoxicity-related factors were significantly increased in the Ara-C and plerixafor dual-injected group in leukemia. To further examine whether immune cell inhibition by CXCR4 could diminish the number of leukemic blasts, we investigated CD3 leukocytes and NK cells, which express CXCR4 in functional cells distributed in the spleen and BM. PB cells from the Ara-C- and plerixafor-only groups and the $\mathrm{P}+\mathrm{A}$ group were isolated and subjected to FACS analysis. The untreated C1498 injection only group and plerixafor treated group showed that CD4 and CD8 cells gradually decreased in a day-dependent manner, implying that plerixafor cannot independently alter the frequency of immune cells. However, the frequency of CD4 and CD8 cells was maintained in the $\mathrm{P}+\mathrm{A}$ group, compared to the Ara-C-only and C1498 injected groups (Fig. 4A). To examine their functional capacity, real-time PCR was performed using primers for $I F N-\gamma$ and cytotoxic-related factors. PCR results revealed a significant upregulation of $I F N-\gamma$ expression and cytotoxic-related factors including perforin and granzyme $B$. $I F N-\gamma$, a main cytokine produced by cytotoxic T and NK cells, can help to kill target cells by immune system activation. As shown in Fig. 4B, the expression levels of IFN- $\gamma$, perforin, and granzyme $B$ in the spleens of the $\mathrm{P}+\mathrm{A}$ group were significantly increased, compared to those of the control (untreated C1498 injection only) group and the plerixafor only group. These results showed high level of genes in $\mathrm{P}+\mathrm{A}$ group, compared to leukemia and single treated group (for $I F N-\gamma ; \mathrm{C} 1498$ injected group vs. 28.0-fold, plerixafor vs. 17.7-fold, Ara-C vs. 2.0-fold, for perforin; C1498 injected group vs. 13.1-fold, plerixafor vs. 3.6-fold, Ara-C vs. 1.2-fold, for granzyme B; C1498 injected group vs. 6.0-fold, plerixafor vs. 3.9-fold), suggesting transcriptional activation in response to leukemic blasts (Fig. 4B). With the Ara-C treated group, a significant difference between the treated and $\mathrm{P}+\mathrm{A}$ groups in expression of the IFN- $\gamma$ and perforin gene, but not granzyme $B$, was detected. In protein level of spleen, high level of $I F N-\gamma$ was detected in $\mathrm{P}+\mathrm{A}$ group, compared to control, Ara-C- and plerixafor-only groups (Fig. 4C and D). Taken together, those results imply a role of functional relevance in immune cells for plerixafor, especially when combined with Ara-C.

\section{Discussion}

Previous studies suggested that CXCR4 is directly involved in tumor promotion and clinical outcomes $(19,20)$. CXCR4 modulation is regarded as a promising strategy to eliminate tumor promotion in tumor environments as well as at the cellular level. Although many studies have shown the effect of plerixafor, the role of CXCR4 in the immune system still remains unclear. To test the antitumoral effects of plerixafor, we investigated whether plerixafor contributed to the suppression of leukemic cells both in vitro and in vivo. In contrast to our expectation, no direct antitumoral effect was found in vitro, 


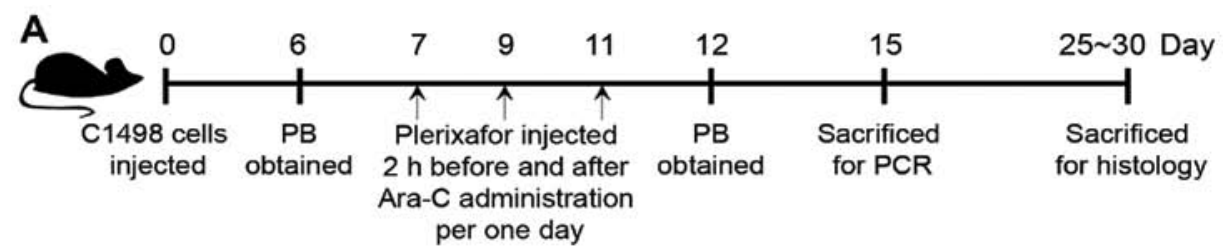

B
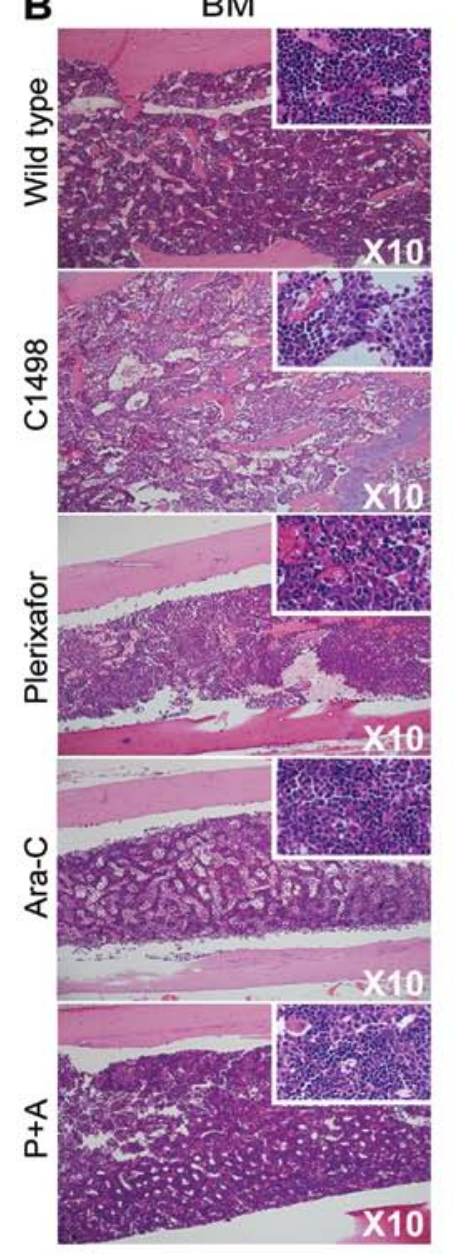

C

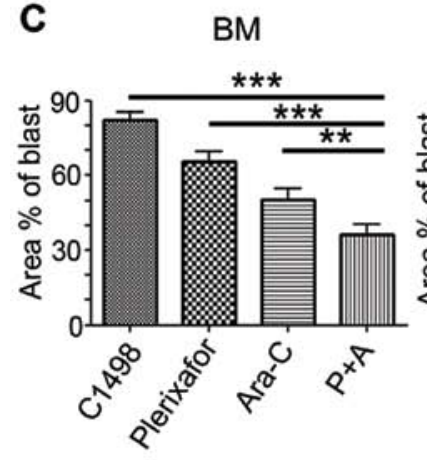

\section{Liver}

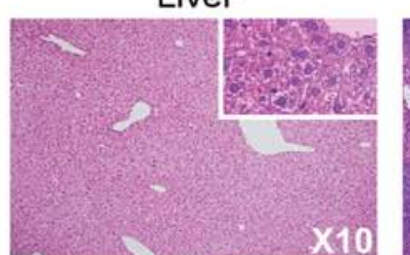

$\times 10$
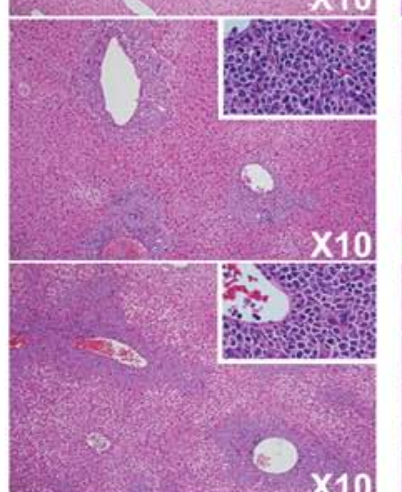

$\times 10$

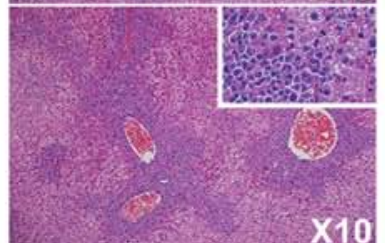

$\times 10$

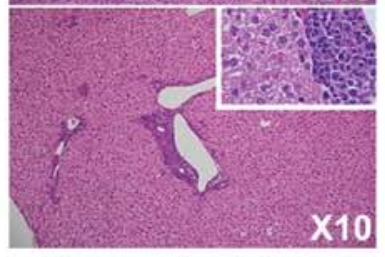

$\times 10$

\section{Spleen}
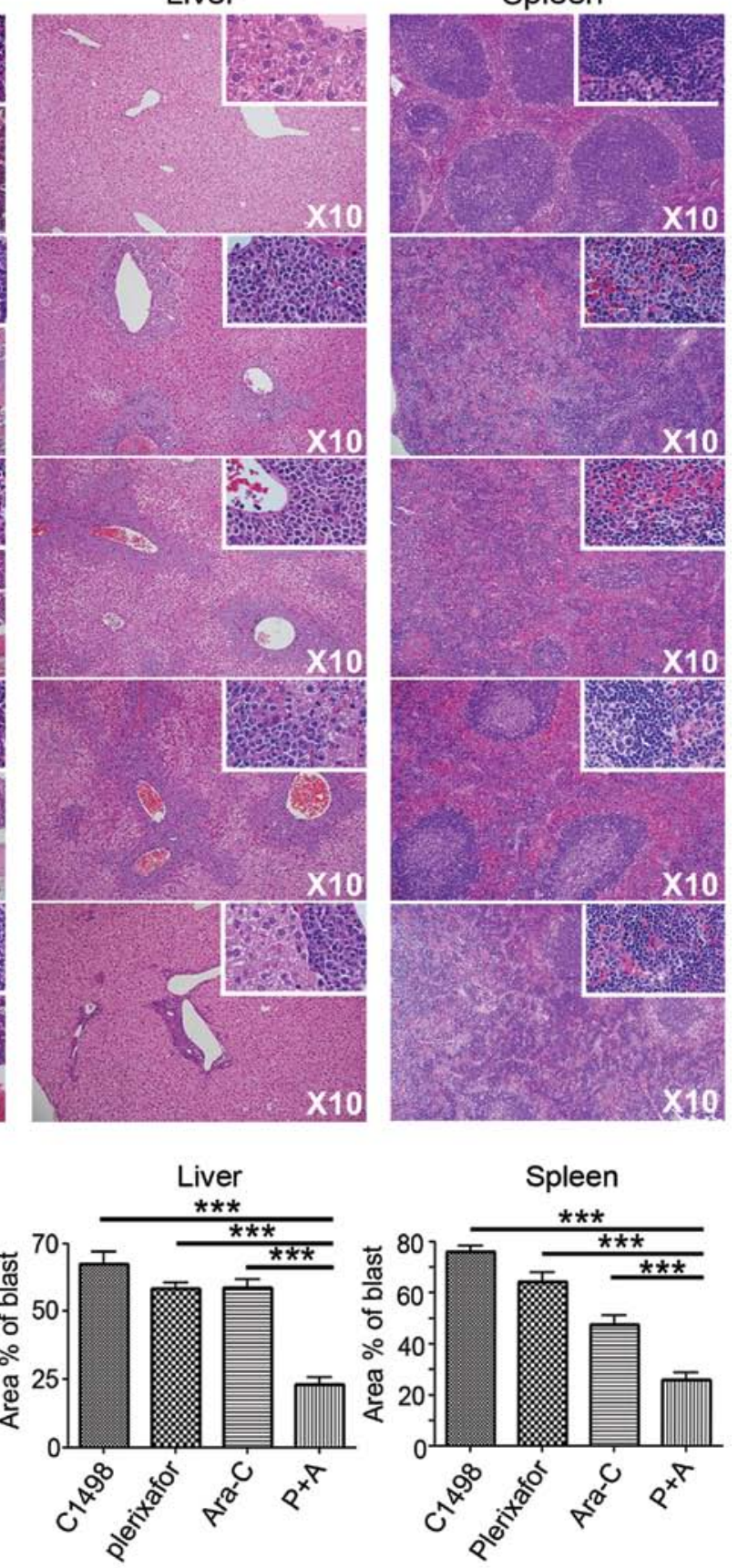

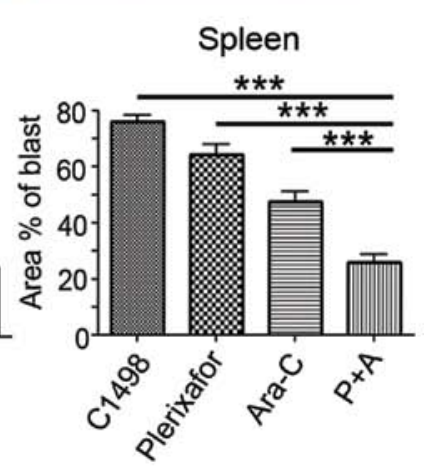

Figure 3. Plerixafor with Ara-C synergistically suppresses the leukemic blasts in vivo. (A) Schematic diagram of the in vivo study. (B) The sections show infiltrated leukemic blasts in tissues including BM, liver, and spleen. Panels clearly show suppression of C1498 infiltration in the P+A group, compared to other groups. Insets (magnification, $\mathrm{x} 40$ ) show enlargement of cells within the main images (magnification, x10). (C) Statistical analysis for panel B. Bars represents mean $\pm \mathrm{SE}$, and the asterisks depict statistically significant differences compared to the $\mathrm{P}+\mathrm{A}$ group $\left({ }^{* *} \mathrm{P}<0.01 ;{ }^{* * *} \mathrm{P}<0.001\right)$.

regardless of whether the treatment was plerixafor alone or in combination with Ara-C. However, a remarkable suppression of leukemic blasts in vivo was detected in the $\mathrm{P}+\mathrm{A}$ combination group, suggesting an unknown role for CXCR4 in the tumor microenvironment. Direct killing from chemotherapy and radiotherapy or through immune cells such as cytotoxic T cells and NK cells is usually regarded as the main strategy to eliminate tumor cells. Because there was no effective suppression of tumor cells in vitro but effective inhibition of blasts in vivo, we postulate that plerixafor has indirect effects, and 

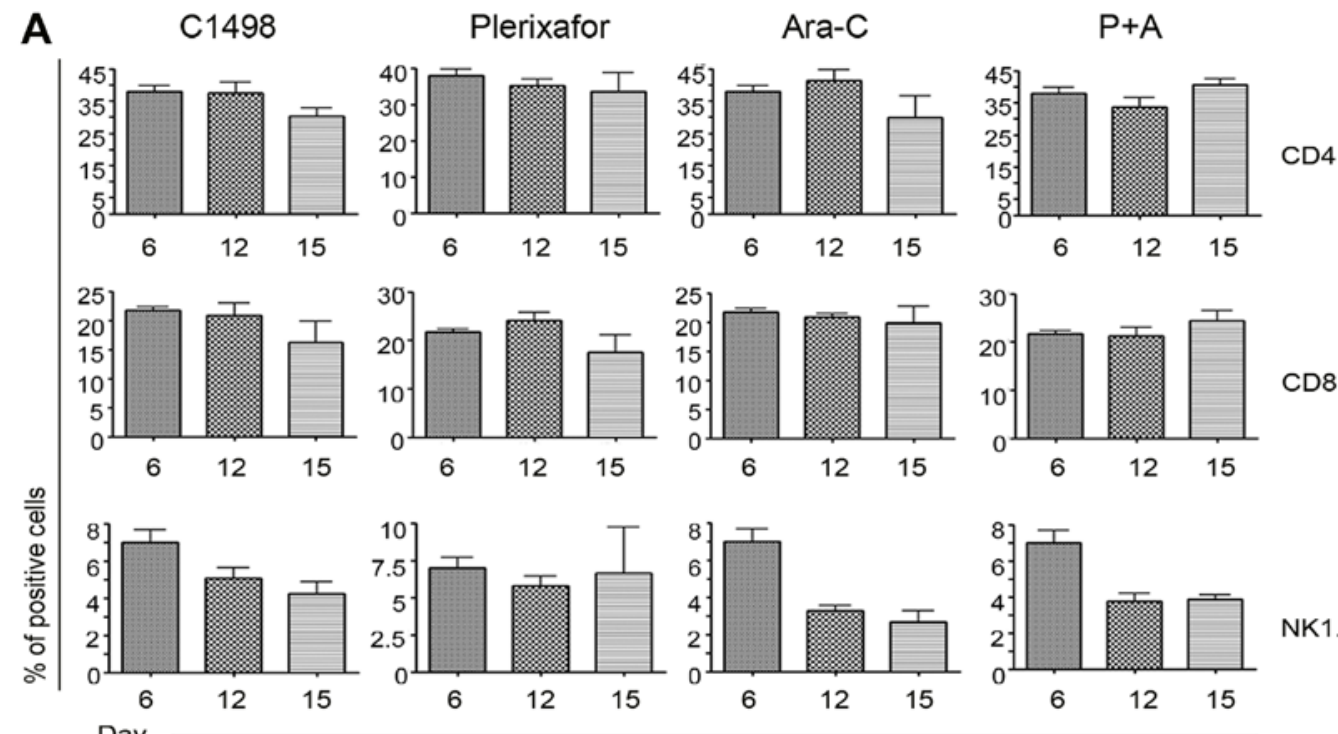

NK1.1
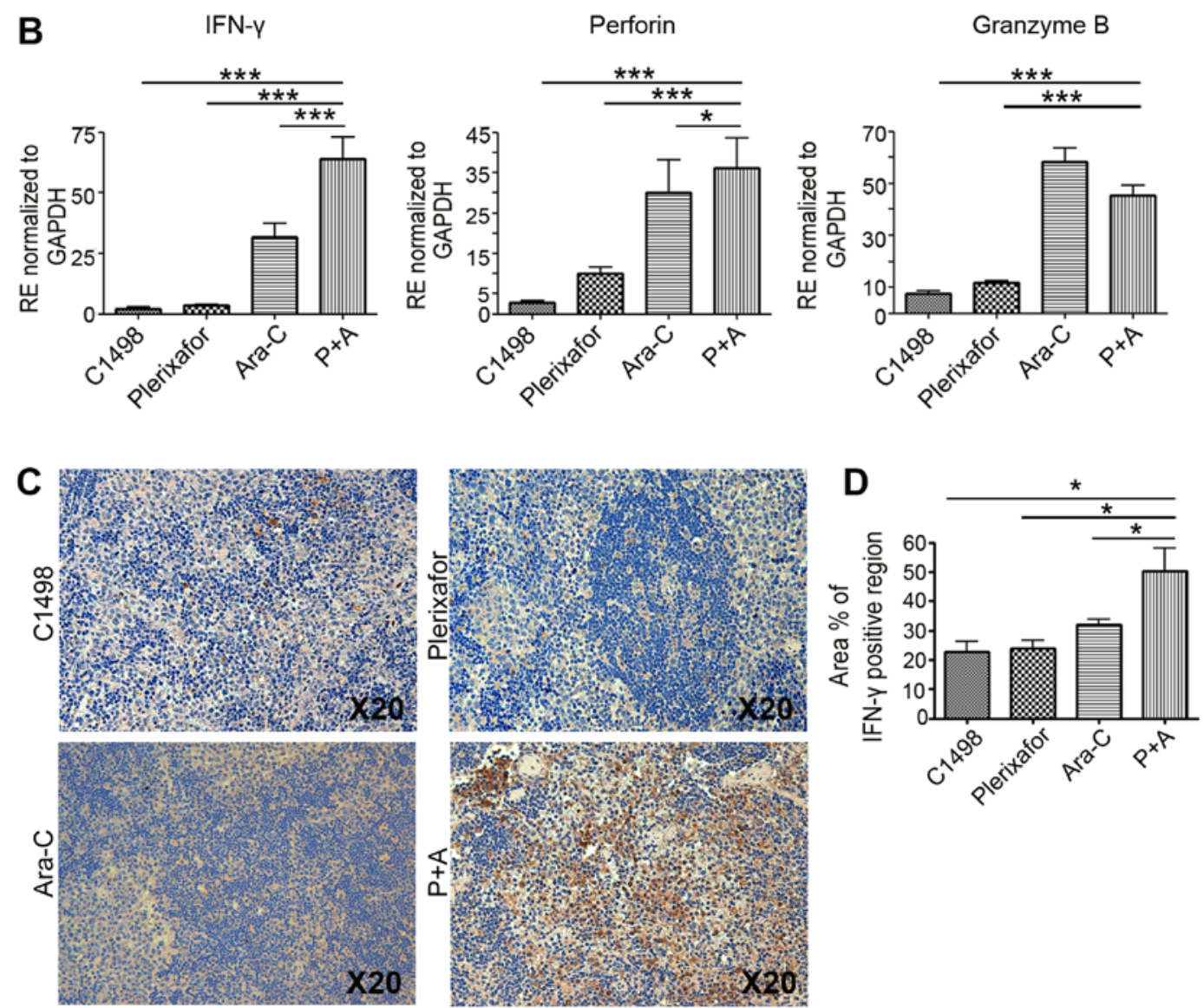

Figure 4. Functional enhancement of immune cells in the plerixafor and Ara-C combination group. (A) Although most immune cells displayed a day-dependent decrease, the frequency of immune cells did not decrease in the P+A group. However, a significant difference was not detected. (B) Activated immune cells in the spleen were analyzed by RT-qPCR. Gene expression was normalized to that of GAPDH. Values represent the mean \pm SE of three independent experiments, each of which was performed in triplicate. ( $\left.\mathrm{P}<0.05 ;{ }^{* * *} \mathrm{P}<0.001\right)$. (C) High level of $I F N-\gamma$ in $\mathrm{P}+\mathrm{A}$ group was detected, compared to other groups (magnification, $\mathrm{x} 20$ ). (D) Statistical analysis using Image $\mathrm{J}$ threshold for panel C. Bars represents mean \pm SE, and asterisks depict statistically significant differences compared to the $\mathrm{P}+\mathrm{A}$ group $(\mathrm{P}<0.05)$.

that it can trigger factors such as immune cells to support the decrease in blasts.

Recently, de Oliveira et al reported the correlation between $I F N-\gamma$ and high CXCR4 expression in immunopathogenesis and suggested that $I F N-\gamma$ induces high levels of CXCR4 and its ligand, resulting in the migration of tumor cells and metastasis, but not exerting antitumor effects (21). In contrast, our data showed a high level of $I F N-\gamma$ mRNA expression in the $\mathrm{P}+\mathrm{A}$ group. Moreover, cytotoxic factors, perforin and granzyme $B$, were highly expressed in the $\mathrm{P}+\mathrm{A}$ group, and 
their expression was significantly different from that of the C1498 injection only group as a control and plerixafor injection only group, suggesting enhanced killing of immune cells by inhibition of CXCR4 with chemotherapy in leukemia. The function and frequency of immune cells and the level of $I F N-\gamma$ overall has been shown to be decreased in leukemia (17). To exclude pathologic conditions, we performed a FACS analysis using normal mononuclear cells (MNCs) with the Ara-C and plerixafor combination treatment in vitro and examined the expression of IFN- $\gamma$, perforin, and granzyme $B$. However, no significant difference in immune cell activation was detected in the P+A group (data not shown), implying that immune activation by CXCR4 inhibition may require another mediator in vivo. For a better understanding of the correlation between CXCR4 and immune cells, further functional studies on the precise mechanism induced at the immune cell level as well as in tissues should be undertaken. Niche-focused in vivo studies of CXCR4 and immune cells are needed to clarify this interaction in leukemia.

The BMM is important for the progression of AML and maintenance of minimal residual disease through soluble factors that are associated with leukemic cell resistance to chemotherapy (22). The centrally located vascular niche of BM is a site that induces differentiation and eventually mobilization of hematopoietic cells to the peripheral blood (23). Remarkably, mature megakaryocytes are located adjacent to sinusoidal endothelial cells (SEC) within BM and migrate to the peripheral circulation through SEC $(24,25)$. We also found high numbers of megakaryocytes around the SEC and decreased circulating leukemic blasts inside the BM sinusoidal capillary (data not shown). In addition, the SEC capillary morphology returned to that of wild-type when plerixafor was injected together with Ara-C (data not shown). This suggests that plerixafor can also support the reconstitution of BM architecture with conventional chemotherapy. While this is not directly relevant to leukemia elimination, we cannot rule out the possibility that niche rearrangement by plerixafor can inhibit engraftment of leukemia.

Not all pathological conditions need proper CXCR4 expression to develop and activate lymphocytes. In WHIM (warts, hypogammaglobulinemia, infections, and myelokathexis) syndrome with rare combined immunodeficiency disorder in BM, a strong response to CXCL12 and displayed chronic non-cyclic leukopenia was shown. Specifically, in the case of $\mathrm{CXCR}^{+/ 1013}$ mutant mice presenting WHIM syndrome, CXCR4 desensitization with plerixafor reversed lymphoneutropenia that had disturbed leukocyte homeostasis (26). This indicates that the microenvironment is governed by diverse pathologic conditions that cause it to differ from an in vitro system.

In summary, we provide clues for the CXCR4 function accompanied with high level of cytotoxicity related factors in leukemic microenvironments that may suggest an advanced therapeutic strategy for leukemia through the modulation of immune cells or their niche by plerixafor.

\section{Acknowledgements}

This study was supported by the Basic Science Research Program through the National Research Foundation of Korea (NRF) funded by the Ministry of Education (2014R1A1A2053407).

\section{References}

1. Löwenberg B, Downing JR and Burnett A: Acute myeloid leukemia. N Engl J Med 341: 1051-1062, 1999.

2. Liesveld JL, Bechelli J, Rosell K, Lu C, Bridger G, Phillips G II and Abboud CN: Effects of AMD3100 on transmigration and survival of acute myelogenous leukemia cells. Leuk Res 31: 1553-1563, 2007.

3. Lane SW, Wang YJ, Lo Celso C, Ragu C, Bullinger L, Sykes SM, Ferraro F, Shterental S, Lin CP, Gilliland DG, et al: Differential niche and Wnt requirements during acute myeloid leukemia progression. Blood 118: 2849-2856, 2011.

4. Krause DS, Fulzele K, Catic A, Sun CC, Dombkowski D, Hurley MP, Lezeau S, Attar E, Wu JY, Lin HY, et al: Differential regulation of myeloid leukemias by the bone marrow microenvironment. Nat Med 19: 1513-1517, 2013.

5. Moshaver B, van der Pol MA, Westra AH, Ossenkoppele GJ Zweegman S and Schuurhuis GJ: Chemotherapeutic treatment of bone marrow stromal cells strongly affects their protective effect on acute myeloid leukemia cell survival. Leuk Lymphoma 49: 134-148, 2008.

6. Konopleva M, Konoplev S, Hu W, Zaritskey AY, Afanasiev BV and Andreeff M: Stromal cells prevent apoptosis of AML cells by up-regulation of anti-apoptotic proteins. Leukemia 16: 1713-1724, 2002.

7. Mercier FE, Ragu C and Scadden DT: The bone marrow at the crossroads of blood and immunity. Nat Rev Immunol 12: 49-60, 2012.

8. Peled A and Tavor S: Role of CXCR4 in the pathogenesis of acute myeloid leukemia. Theranostics 3: 34-39, 2013.

9. Tokoyoda K, Egawa T, Sugiyama T, Choi BI and Nagasawa T: Cellular niches controlling B lymphocyte behavior within bone marrow during development. Immunity 20: 707-718, 2004.

10. Foudi A, Jarrier P, Zhang Y, Wittner M, Geay JF, Lecluse Y, Nagasawa T, Vainchenker W and Louache F: Reduced retention of radioprotective hematopoietic cells within the bone marrow microenvironment in CXCR $4^{-/}$chimeric mice. Blood 107 : 2243-2251, 2006.

11. Fricker SP, Anastassov V, Cox J, Darkes MC, Grujic O, Idzan SR, Labrecque J, Lau G, Mosi RM, Nelson KL, et al: Characterization of the molecular pharmacology of AMD3100: A specific antagonist of the G-protein coupled chemokine receptor, CXCR4. Biochem Pharmacol 72: 588-596, 2006.

12. Burger JA and Peled A: CXCR4 antagonists: Targeting the microenvironment in leukemia and other cancers. Leukemia 23: 43-52, 2009.

13. Zhang Y, Patel S, Abdelouahab H, Wittner M, Willekens C, Shen S, Betems A, Joulin V, Opolon P, Bawa O, et al: CXCR4 inhibitors selectively eliminate CXCR4-expressing human acute myeloid leukemia cells in NOG mouse model. Cell Death Dis 3: e396, 2012.

14. Zeng Z, Shi YX, Samudio IJ, Wang RY, Ling X, Frolova O, Levis M, Rubin JB, Negrin RR, Estey EH, et al: Targeting the leukemia microenvironment by CXCR4 inhibition overcomes resistance to kinase inhibitors and chemotherapy in AML. Blood 113: 6215-6224, 2009.

15. Sison EA, McIntyre E, Magoon D and Brown P: Dynamic chemotherapy-induced upregulation of CXCR4 expression: A mechanism of therapeutic resistance in pediatric AML. Mol Cancer Res 11: 1004-1016, 2013.

16. Nervi B, Ramirez P, Rettig MP, Uy GL, Holt MS, Ritchey JK Prior JL, Piwnica-Worms D, Bridger G, Ley TJ, et al: Chemosensitization of acute myeloid leukemia (AML) following mobilization by the CXCR4 antagonist AMD3100. Blood 113: 6206-6214, 2009.

17. Lee JY, Park S, Min WS and Kim HJ: Restoration of natural killer cell cytotoxicity by VEGFR-3 inhibition in myelogenous leukemia. Cancer Lett 354: 281-289, 2014.

18. Kremer KN, Peterson KL, Schneider PA, Meng XW, Dai H, Hess AD, Smith BD, Rodriguez-Ramirez C, Karp JE, Kaufmann $\mathrm{SH}$, et al: CXCR4 chemokine receptor signaling induces apoptosis in acute myeloid leukemia cells via regulation of the Bcl-2 family members Bcl-XL, Noxa, and Bak. J Biol Chem 288: 22899-22914, 2013.

19. Mannelli F, Cutini I, Gianfaldoni G, Bencini S, Scappini B, Pancani F, Ponziani V, Bonetti MI, Biagiotti C, Longo G, et al: CXCR4 expression accounts for clinical phenotype and outcome in acute myeloid leukemia. Cytometry B Clin Cytom 86: 340-349, 2014. 
20. Müller A, Homey B, Soto H, Ge N, Catron D, Buchanan ME, McClanahan T, Murphy E, Yuan W, Wagner SN, et al: Involvement of chemokine receptors in breast cancer metastasis. Nature 410: 50-56, 2001.

21. de Oliveira KB, Guembarovski RL, Guembarovski AM, da Silva do Amaral Herrera AC, Sobrinho WJ, Ariza CB and Watanabe MA: CXCL12, CXCR4 and IFN $\gamma$ genes expression: Implications for proinflammatory microenvironment of breast cancer. Clin Exp Med 13: 211-219, 2013.

22. Meads MB, Hazlehurst LA and Dalton WS: The bone marrow microenvironment as a tumor sanctuary and contributor to drug resistance. Clin Cancer Res 14: 2519-2526, 2008.

23. Kopp HG, Avecilla ST, Hooper AT and Rafii S: The bone marrow vascular niche: Home of HSC differentiation and mobilization. Physiology (Bethesda) 20: 349-356, 2005.
24. Psaila B, Lyden D and Roberts I: Megakaryocytes, malignancy and bone marrow vascular niches. J Thromb Haemost 10: 177-188, 2012.

25. Yin $\mathrm{T}$ and $\mathrm{Li} \mathrm{L}$ : The stem cell niches in bone. J Clin Invest 116 : 1195-1201, 2006.

26. Balabanian K, Brotin E, Biajoux V, Bouchet-Delbos L, Lainey E, Fenneteau O, Bonnet D, Fiette L, Emilie D and Bachelerie F: Proper desensitization of CXCR4 is required for lymphocyte development and peripheral compartmentalization in mice. Blood 119: 5722-5730, 2012. 